\title{
Notas Farmacológicas \\ El rol de los antioxidantes en la prevención de enfermedades cardiovasculares
}

\section{Introducción}

Los factores de riesgo clásicos no resultan totalmente suficientes para explicar porqué algunos individuos desarrollan enfermedad cardiovascular y otros no. En función de ello, existe (desde hace más de 20 años) gran interés en la identificación de nuevos factores de riesgo cuya modificación podría resultar en importantes beneficios para la salud.

Evidencia originada en investigación básica sugiere diversos mecanismos biológicamente plausibles en la producción de enfermedad cardiovascular. Quizás uno de los universalmente aceptados es que la peroxidación de ciertos lípidos plasmáticos (como las LDL) favorece su depósito subendotelial y la formación ulterior de placas de ateroma. Esta teoría (comprobada experimentalmente en modelos animales) dio origen a la hipótesis de que ciertas sustancias reductoras con capacidad de prevenir o revertir la oxidación de las LDL podrían evitar el desarrollo ulterior de enfermedad cardiovascular.

\section{Rol de los antioxidantes y estudios observacionales: resultados} prometedores

La hipótesis biológica se ve reforzada por estudios epidemiológicos observacionales que han encontrado, en forma consistente, ${ }^{1-3}$ una relación inversa entre morbimortalidad cardiovascular y ciertos hábitos dietarios (alta proporción de alimentos ricos en antioxidantes) o la ingesta de suplementos vitamínicos antioxidantes (vitamina $\mathrm{E}$ [de-tocoferol]).

En varios estudios de cohortes 4 , la magnitud del "efecto protector cardiovascular" observado es sumamente importante: en el caso de la vitamina $E$, estudios epidemiológicos prospectivos encontraron que su ingestión diaria por un periodo mayor a dos años, se asoció a una disminución de entre un 31 a un $65 \% 4$ del riesgo relativo* de diversos eventos cardiovasculares.

Este aparente beneficio (observado tanto con las dietas ricas en antioxidantes como con los suplementos de vitamina purificada ${ }^{5}$ ) debe ser interpretado con extrema cautela.

La razón es sencilla: los estudios observacionales tienen limitaciones metodológicas que les impiden descartar que los efectos beneficiosos observados puedan deberse a razones distintas a la dieta o la suplementación vitamínica. Básicamente, puede ocurrir que el grupo compuesto por quienes han decidido seguir las dietas o ingerir las vitaminas difiera, además, en otros factores pronósticos adicionales de la población tomada como control en los estudios. Es decir: además de una diferente dieta y/o ingesta de vitaminas, puede ser que los grupos tengan diferente nivel de actividad física, distinto nivel socioeconómi- co, etc. De existir estas diferencias en factores de riesgo cardiovascular (¡algo que no puede descartarse!) se constituiría un sesgo de selección*. Esta Limitación impide realizar inferencias causales con estudios observacionales.

La mejor manera de evitar sesgos es la realización de ensayos clínicos aleatorizados. En ellos, entre otras medidas para controlar sesgos, se busca que el azar distribuya en forma balanceada los factores pronósticos, dando origen a dos grupos idealmente idénticos entre sí y que diferirán exclusivamente en la variable de análisis. Por ejemplo: si tomaron o no suplemento de vitamina $E$. Si se asume que los grupos se encuentran balanceados respecto a todos los factores de riesgo conocidos (y especialmente respecto a los no conocidos), cualquier eventual diferencia de morbimortalidad podría ser en principio atribuida a la exposición experimental a la vitamina $\mathrm{E}$.

\section{Impacto del efecto}

La relevancia de una investigación clínica en el área no es menor: 1. Intenta verificar la evidencia previa que sugiere una disminución de riesgo potencialmente importante ( 31 al $65 \%$ en estudios de cohortes) en enfermedades de alta prevalencia.

2. Ensaya una medida terapéutica de costo relativamente bajo para los sistemas de salud, autoadministrable por la población general (por ejemplo, un suplèmento de vitamina E).

3. Utiliza una intervención de baja toxicidad para evitar una patología de elevada morbimortalidad.

Con la excepción de los ensayos clínicos de vacunas, difícilmente exista a priori un escenario superior en cuanto al cociente riesgo/beneficio de la prevención de enfermedades cardiovasculares con una vitamina antioxidante (o dietas ricas en ellas). En función de lo anterior, comenzaron a llevarse a cabo varios ensayos clínicos aleatorizados. Todos buscaban testear experimentalmente una hipótesis similar: "la vitamina $\mathrm{E}$ (inhibiendo la peroxidación de las LDL) podría causar una reducción de la morbimortalidad cardiovascular".

Evidencia grado $1^{*}$ : efectos reales de la vitamina E en prevención primaria y secundaria cardiovascular.

Hasta la fecha, cinco de los ensayos clínicos que evaluaban vitamina $E$ en enfermadades cardiovasculares han finalizado y publicado sus resultados. Suman en total más de 80.000 sujetos y más de 416.000 personas/año de seguimiento.

En la siguiente tabla se agrupan algunos datos, para permitir una apreciación rápida de sus características fundamentales.

\begin{tabular}{|c|c|c|c|c|c|c|}
\hline Ensayo clínico & Población & Seguimiento & Dosis de vitamina $\mathbf{E}$ & Resultados & \% reducción $\mathrm{RR}^{*}$ & IC $95 \% *$ \\
\hline \multirow[t]{2}{*}{$\begin{array}{c}\text { China } 6 \\
1993\end{array}$} & $\begin{array}{l}29.584 \text { adultos. } \\
\text { Provincia de Linxian }\end{array}$ & 5,2 años & $30 \mathrm{mg} /$ día + selenio + bcaroteno & Mortalidad total & 9 & 0 a 17 (NS) \\
\hline & & & & Mortalidad $\times \mathrm{ACV}$ & 9 & -8 a 24 (NS) \\
\hline \multirow[t]{3}{*}{$\begin{array}{c}\text { ATBC }^{7} \\
1994-97-98 \\
\end{array}$} & $\begin{array}{l}29.133 \text { varones fu- } \\
\text { madores. Finlandia }\end{array}$ & 6.1 años & $50 \mathrm{mg} / \mathrm{día}$ & Mortalidad total & -2 & -9 a5 (NS) \\
\hline & & & & Muerte CV & 2 & -8 a 11 (NS) \\
\hline & & & & Angor & 9 & $1 \mathrm{a} 17$ (NS) \\
\hline \multirow[t]{3}{*}{$\begin{array}{c}\text { CHAOS }^{8} \\
1996\end{array}$} & $\begin{array}{l}2.002 \text { pacientes con } \\
\text { enfermedad corona- } \\
\text { ria. East Anglia }\end{array}$ & 1,4 años & 400 u 800 UI/ día & Muerte CV, IAM no fatal & 47 & 17 a $66(S)$ \\
\hline & & & & IAM no fatal & 77 & 53 a $89(S)$ \\
\hline & & & & Mortalidad total & -29 & $-119 \mathrm{a} 24$ (NS) \\
\hline \multirow[t]{2}{*}{$\begin{array}{c}\text { GISSI - P } 9 \\
1999\end{array}$} & $\begin{array}{l}11.324 \text { pacientes so- } \\
\text { brevivientes IAM re- } \\
\text { ciente. Italia }\end{array}$ & 3,5 años & $300 \mathrm{mg} /$ día & $\begin{array}{l}\text { Muerte, IAM o ACV no } \\
\text { fatales }\end{array}$ & 11 & -3 a 23 (NS) \\
\hline & & & & $\begin{array}{l}\text { Muerte CV, IAM o ACV no } \\
\text { fatales }\end{array}$ & 12 & -4 a 25 (NS) \\
\hline \multirow[t]{2}{*}{$\begin{array}{l}\text { HOPE } 10 \\
2000\end{array}$} & $\begin{array}{l}9.541 \text { pacientes de } \\
\text { riesgo CV. Canadá }\end{array}$ & 4,5 años & $400 \mathrm{UI} / \mathrm{dia}$ & Muerte CV, IAM o ACV & -5 & -16 a 5 (NS) \\
\hline & & & & Mortalidad total & 0 & -13 a11 (NS) \\
\hline
\end{tabular}


Como se observa en la tabla, casi todos los estudios no observaron efectos benéficos de la vitamina E. El único estudio clínico aleatorizado que arrojó resultados positivos para la intervención (suplementación de vitamina E) en la prevención de enfermedades cardiovasculares fue el Cambridge Heart Antioxidants Study, realizado en el Reino Unido y publicado en 1996. En el mismo, se verificó una reducción estadísticamente significativa del $47 \%$ del resultado final primario del estudio: el punto final combinado de muerte cardiovascular e infarto de miocardio no fatal. Esta reducción se debió enteramente a una reducción del riesgo relativo del $77 \%$ en la ocurrencia de IAM no fatales. Sin embargo, varios problemas metodológicos fueron detectados en este estudio, lo cual cuestiona la validez interna* del CHAOS y por ello sus resultados se consideran no concluyentes. ${ }^{11}$

\section{Ejemplos:}

- El número de eventos totales fue bajo respecto al esperado. Esta situación puede no invalidar los resultados pero sin duda aumenta la posibilidad de que las diferencias observadas se deban a un extremo juego del azar.

- No existe consistencia entre los resultados del estudio y el resto de la evidencia disponible: el efecto es exageradamente mayor que el esperable y se produce en un intervalo de tiempo inferior. El efecto de la vitamina $\mathrm{E}$ es superior al del control de factores de riesgo tradicionales (tales como el tratamiento de la hipertensión arterial o el efecto logrado con las estatinas en el control de la hipercolesterolemia).

- Al realizar análisis de subgrupos con los datos del CHAOS, otros autores 12 encuentran un incremento "estadísticamente significativo" en la mortalidad temprana por enfermedad coronaria en el grupo tratado con vitamina $\mathrm{E}$. En función de ello, sostienen que el incremento "estadísticamente no significativo" en la mortalidad total observado en el estudio no debería ser dejado de lado.

- Aún asumiendo que los cinco estudios tuvieran igual calidad metodológica, debería primar el sentido común: un estudio de 2800 personas/año de seguimiento se opone en sus conclusiones a cuatro estudios que suman 414.000 personas/año de seguimiento.
Diversas hipótesis se tejen respecto a la causa de las diferencias observadas entre los distintos ensayos clínicos ya realizados. Una de las más destacadas es el argumento de diferencias genéticas entre las poblaciones del CHAOS y las de GISSI-P. Otras teorías llegan a cuestionar de plano la plausibilidad biológica de la utilidad de los antioxidantes:

Las células humanas funcionan habitualmente en un estado reducido, que se manifiesta por un cociente elevado entre las formas GSH/GSSG de glutation.

Sin embargo, múltiples procesos celulares precisan oxidación localizada en forma transitoria para Llevarse a cabo: la estructura cuaternaria de las proteínas, el ciclo celular, la apoptosis e incluso la propia adaptación al daño oxidativo ${ }^{13}$. Básicamente, se postula que el elevado poder reductor de las dosis farmacológicas de antioxidantes inhibe múltiples procesos citoplasmáticos normales, y es por lo tanto capaz de generar daño celular per se.

\section{¿Destierro definitivo de la Vitamina E?}

Aunque la administración masiva a nivel poblacional no parece ser efectiva, aun se plantean dudas acerca de su potencial utilidad en poblaciones seleccionadas. Un problema que está surgiendo es que en todos los estudios realizados no se midió el "estado oxidativo" de la población (se mide a través de la tasa de peroxidación de LDL). Tampoco se evaluó si la vitamina E alteraba esta tasa y en qué medida. Por lo tanto es probable que en el futuro nos encontremos con estudios de antioxidantes, pero en el grupo de personas más "oxidadas". Aun falta escribir el último capítulo de esta historia.

\section{Concluyendo}

No hay hasta el momento resultados que avalen el uso de la vitamina E como una medida preventiva primaria o secundaria para la disminución del riesgo de enfermedades cardiovasculares.

El objetivo de las medidas de intervención debe seguir siendo el control de los factores de riesgo conocidos.

*Ver glosario

Dr. Fernando Colmenero Médico de Familia. Centro de Estudios Farmacoeconómicos . 19 de mayo de 2000

\section{Bibliografía}

1. Stampfer MJ, Hennekens CH, Mansosn JE, Colditz GA, Rosner B, Willet WC Vitamin E consumption and the risk of coronary disease in women. N EngL J Med 1993, May 20; $328(20): 1444-9$

2. Rimm EB, Stampfer MJ, Ascherio A, Giovannucci E, Colditz GA, Willet WC Vitamin E consumption and the risk of coronary heart disease in men. N Engl J Med 1993 May 20; $328(20): 1450-6$

3. Kushi LH, Folsom AR, Prineas RJ, Mink PJ, Wu Y, Bostick RM Dietary antioxidant vitamin and death from coronary heart disease in postmenopausal women. N Engl J Med 1996 May 2; 334 (18): 1156-62

4. Jha P, Flather M, Lonn E, Farkouh M, Yusuf S The antioxidant vitamins and cardiovascular disease: a critical review of epidemiologic and clinical trials data. Ann Int Medicine 1995, 123 (11) 860-872

5. Lonn E M. Yusuf S Is there a role for antioxidant vitamins in the prevention of cardiovascular diseases: an update on epidemiological and clinical trials data. Can J Cardiology 1997, $13(10), 957-965$

6. Blot WJ, Li JY, Guo W, Dawsey S Nutrition intervention trials in Linxian, China: supplementation with specific vitamin/mineral combinations, cancer incidence, and disease specific mortality in the general population. J Natl Cancer Inst 1993; 1483-92

7. The effecto of vitamin $\mathrm{E}$ and beta-carotene on the incidence of lung cancer akn other cancers in male smokers. The Alpha Tocopherol, Beta Carotene Cance Prevention Study. N Engl J Med 1994 Apr 14;330(15):1029:35

Otros estudios relacionados del mismo grupo:

Rapola JM, Virtamo J, Ripatti S, Huttunen JK, Albanes D, Taylor PR, Heinonen OP Randomised trial of alpha-tocopherol and beta-carotene supplements on incidence of major coronary events in men with previous myocardial infarction. Lancet 1997, Jun 14; 349(9067): 1715-20

8. Stehpens NG, Parsons A, Schofield PM, Randomised controlled trial of vitamin E in patients with coronary disease: Cambridge Heart

Antioxidant Study. Lancet 1996 Mar 23:347(9004):781-6

9. Gruppo Italiano per lo Studio della Sopravvivenza nell' infarto miocardico Dietary supplementation with n-3 polyunsaturated fatty acids and vitamin E after myocardial infarction: results of the GISSI-Prevenzion trial. Lancet 1999; 354:447-55

10. The Heart Outcomes Prevention evaluation Study Investigators Vitamin E supplementation and cardiovascular events in high-risk patients N Engl J Med 2000; 342:154-60.

11. Lonn E M, Yusuf S, Emerging approaches in preventing cardiovascular diseases. BMJ 1999; 318:1337-41

12. Ness A, Smith GD Mortality in the CHAOS trial. Lancet 353; 915720 march 1999

13. Halliwell B The antioxidant paradox Lancet 2000, 355: 1179-80 